\title{
Reference X-ray Irradiation System for Personal Dosimeter Testing and Calibration of Radiation
}

\section{Detector}

\author{
Seung Kyu Lee ${ }^{1, *}$, Insu Chang', Sang In Kim², Jungil Lee', Hyoungtaek Kim', Jang-Lyul Kim', Min Chae Kim ${ }^{1,3}$ \\ ${ }^{1}$ Korea Atomic Energy Research Institute, Daejeon, Korea; ${ }^{2}$ ARIM Science, 310 CTCC, Daejeon, Korea; ${ }^{3}$ Department of Nuclear Engineering, \\ Hanyang University, Seoul, Korea
}

\section{Technical Paper}

Received February 20, 2019

Revision May 2, 2019

Accepted May 13, 2019

Corresponding author: Seung Kyu Lee

Korea Atomic Energy Research Institute, 989-111 Daedeok-daero, Yuseong-gu,

Daejeon 34057, Korea

Tel: $+82-42-868-2272$

Fax: +82-42-868-8609

E-mail: sklee11@kaeri.re.kr

This is an Open-Access article distributed under the terms of the Creative Commons Attribution NonCommercial License (http://creativecommons.org/ licenses/by-nc/4.0) which permits unrestricted noncommercial use, distribution, and reproduction in any medium, provided the original work is properly cited.

Copyright @ 2019The Korean Association for Radiation Protection

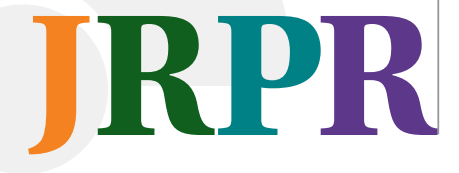

Background: In the calibration and testing laboratory of Korea Atomic Energy Research Institute, the old X-ray generator used for the production of reference X-ray fields was replaced with a new one. For this newly installed X-ray irradiation system, beam alignment as well as the verification of beam qualities was conducted.

Materials and Methods: The existing X-ray generator, Phillips MG325, was replaced with YXLON Y.TU 320-D03 in order to generate reference X-ray fields. Theoretical calculations and Monte Carlo simulations were used to determine initial filter thickness. Beam alignment was performed in three steps to deliver a homogeneous radiation dosage to the target at different distances. Finally, the half-value layers were measured for different X-ray fields to verify beam qualities by using an ion chamber.

Results and Discussion: Beam alignment was performed in three steps, and collimators and other components were arranged to maintain the uniformity of the mean air kerma rate within $\pm 2.5 \%$ at the effective beam diameter of $28 \mathrm{~cm}$. The beam quality was verified by using halfvalue layer measurement methods specified by American National Standard Institute (ANSI) N13.11-2009 and International Organization for Standardization (ISO)-4037. For each of the nine beams than can be generated by the new X-ray irradiation system, air kerma rates for Xray fields of different beam qualifies were measured. The results showed that each air kerma rate and homogeneity coefficient of the first and second half-value layers were within $\pm 5 \%$ of the recommended values in the standard documents.

Conclusion: The results showed that the new X-ray irradiation system provides beam qualities that are as high as moderate beam qualities offered by National Institute of Standards and Technology in ANSI N13.11-2009 and those for narrow-spectrum series of ISO-4037.

Keywords: Reference X-ray field, X-ray generator, Beam alignment, ISO reference beam, NIST reference beam, Half-value layer measurement

\section{Introduction}

Personal dosimeters and survey meters are the instruments used by radiation workers for monitoring and managing radiation dose. Photon energy responses can vary depending on the kind of dosimeters and survey meters used, and for their calibration, a reference radiation field is required. Korea Atomic Energy Research Institute (KAERI) has been designated a national radiation calibration and testing laboratory by the Ko- 
rean government and has established reference radiation fields and a secondary standard dosimetry laboratory in accordance with Korean Industrial Standards (KS) and International Organization for Standardization (ISO) standards [1-3]. To establish infrastructure for national research projects in radiation dosimetry and ensure the reliability and high quality of reference irradiation for personal dosimeter performance testing and the calibration of the radiation detector, it is necessary to design and establish reference radiation fields and conduct beam quality assessment and verification in accordance with domestic and international standards. KAERI built a world-class reference radiation field and calibration and testing system back in 1992 and since then has been conducting research on radiation dosimetry and delivering technical assistance both domestically and internationally. In the reference radiation fields of KAERI, all types of radiation such as gamma, beta, $\mathrm{X}$-rays and neutrons can be used. In particular, the reference X-ray field is designed to produce and use 20 different types of beams including beam qualities offered by National Institute of Standards and Technology (NIST) in American National Standard Institute (ANSI) N13.11-2009 and reference beam qualities by ISO-4037 with the use of an X-ray generator and rotation-type additional filtration system. The old X-ray generators were replaced with new ones, as they caused system instability and decreased output, and additional radiation fields needed to be built in accordance with international standards for testing personal dosimeter performance.

For testing the performance of personal dosimeter readout system and calibrating the radiation detector via X-ray radiation, regular doses of radiation need to be provided at
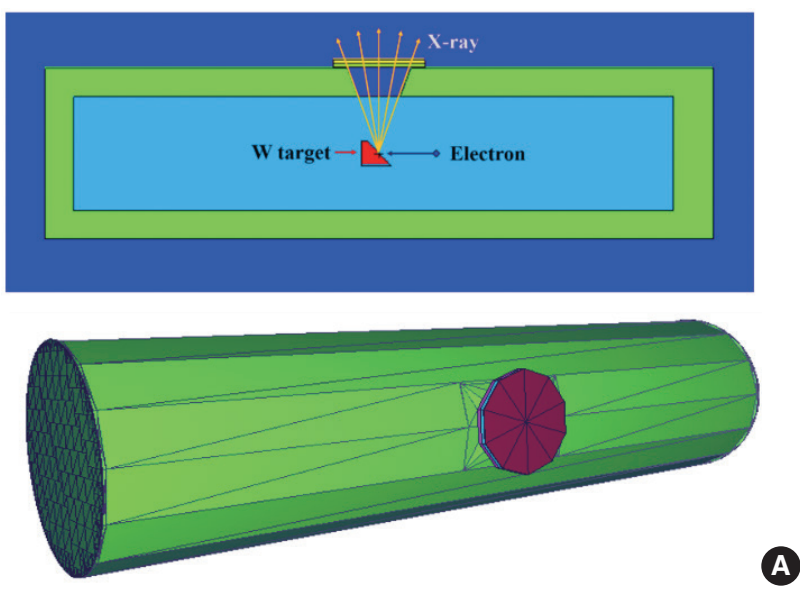

specified positions and therefore, beam alignment should be performed for the new X-ray generators. The measurement of beam uniformity and half-value layer (HVL) of the reference X-ray field should be performed in order to ensure the beam qualities meet the international standards for X-ray fields [1].

In this study, 9 beam qualities of the new X-ray field were assessed, including NIST moderate beam qualities (M-series) and ISO narrow-spectrum (NS-series) beam qualities, and the quality of the reference X-ray field was investigated compared to the international standards of beam qualities.

\section{Materials and Methods}

\section{Establishment of reference X-ray field}

The existing X-ray generator, Phillips MG325, was replaced with YXLON Y.TU 320-D03 to construct reference X-ray fields. This generator has a maximum tube voltage of $320 \mathrm{kV}$ and the voltage can be adjusted by $0.2 \mathrm{kV}$. The tube current can be adjusted by $0.05 \mathrm{~mA}$, up to a maximum of $22.5 \mathrm{~mA}$. At the maximum tube voltage, tube current can be set to a maximum of $13 \mathrm{~mA}$. This generator has a maximum power of 4.2 $\mathrm{kW}$ and is equipped with a 3.0-mm beryllium filter [4]. The irradiator of the X-ray generator opens and closes by an electromagnet, in approximately 0.1 second. The entrance of the $\mathrm{X}$-ray irradiation room and the door to the irradiator are locked by a safety lock to maintain control over radiation sources and immediately respond to any kind of radiation emergency. Laser positioning systems are installed on the wall and the upper part of the irradiator to match the radiation point to the calibration reference point and deliver radi-

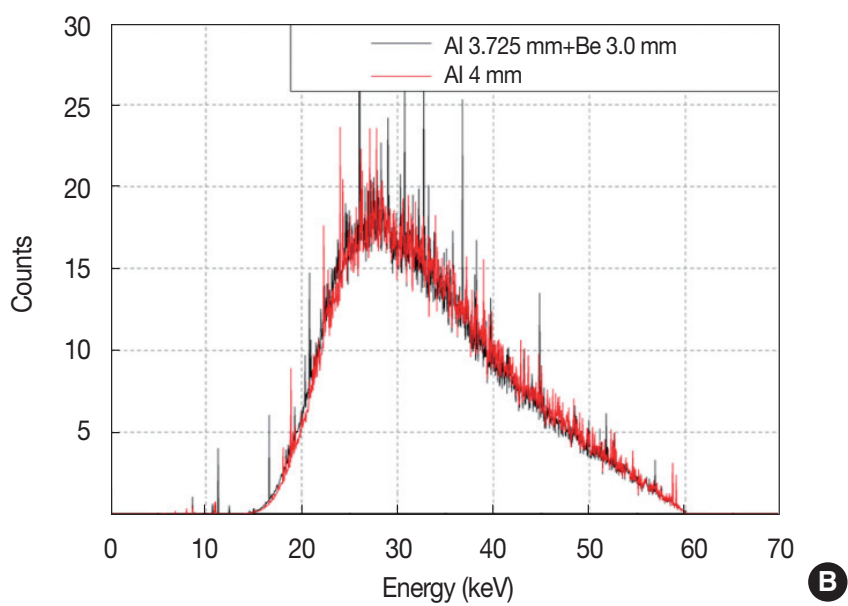

Fig. 1. (A) MCNPX simulation geometry (3D view), and (B) simulation results. 
ation at any point in time.

As the inherent filter, a 3.0-mm beryllium filter is recommended for the X-ray field of NIST M-series with a tube voltage of $60 \mathrm{kV}$ or higher. Filter materials equivalent to a $4.0-\mathrm{mm}$ aluminum filter at the tube voltage of $60 \mathrm{kV}$ are recommended by ISO for the X-ray field with a tube voltage of $60 \mathrm{kV}[1,5]$. The X-ray generator of this study is equipped with a $3.0-\mathrm{mm}$ beryllium window. An additional aluminum filter was installed to use the existing rotation-type additional filtration system. The thickness of the aluminum filter calculated from Equation 1 was $3.725 \mathrm{~mm}$, and this was verified via Monte Carlo simulation. The geometry used in the simulation is shown in Figure 1A. Computer simulations were undertaken for bremsstrahlung X-rays produced by accelerated electrons on a tungsten target. The MCNPX code (2.7.0 version) was used for the simulations and ENDF/B-VI cross-section libraries were used. An F5 detector tally was used for measuring the energy distribution of bremsstrahlung X-rays.

$$
\left(\frac{\mu_{\mathrm{a}}}{\rho}\right)_{\mathrm{Al}} \rho_{\mathrm{Al}} \times t_{A l_{T}}=\left(\frac{\mu_{\mathrm{a}}}{\rho}\right)_{\mathrm{Be}} \rho_{\mathrm{Be}} \times t_{B e}+\left(\frac{\mu_{\mathrm{a}}}{\rho}\right)_{\mathrm{Al}} \rho_{\mathrm{Al}} \times t_{A l},(\text { Eq.1 })
$$

where $\frac{\mu_{a}}{\rho}$ : the mass energy-absorption coefficient $\rho$ : density

$t_{A l_{T}}: 4.0$-mm aluminum filter

$t_{B e}: 3.0$-mm beryllium filter

$t_{A l}$ : additional aluminum filter

All simulations were performed using tube voltages and tube currents. The results of the X-rays were compared with those from the 4-mm aluminum filter, as shown in Figure 1B. The simulation results showed an equivalence between the thickness of the filter installed by KAERI and the 4-mm alu- minum filter in all energy intervals, with a deviation of $\pm 5 \%$.

\section{Beam alignment of $\mathrm{X}$-ray generator}

Reference X-ray fields need to be designed to deliver a uniform dose of radiation at specified positions, in accordance with international standards. Therefore, beam alignment was performed in three steps, which include (1) primary beam alignment using lasers, (2) secondary beam alignment using an X-ray film and densitometer, and (3) tertiary beam alignment using an ion chamber.

First, the primary beam alignment was performed by physical methods using the horizontal and vertical laser positioning system to ensure that the new $\mathrm{X}$-ray generator is aimed at the right angles, both vertically and horizontally, to the target. Physical beam alignment was performed to check whether the laser beam passes through the pinhole collimator and constantly points towards the window of the X-ray generator, by varying the collimator's distances.

The secondary beam alignment was performed using an $\mathrm{X}$-ray film and densitometer. An analysis was conducted on the X-ray images that were obtained at $100-\mathrm{cm}$ and $200-\mathrm{cm}$ distances from the focal point of the X-ray generator. The $\mathrm{x}$ ray film cassette has a metal part with a pinhole at its center, which can determine whether the beam moves vertically. Figure $2 \mathrm{~A}$ is the picture of the experimental setup for the secondary beam alignment using an X-ray film and densitometer. Figure $2 \mathrm{~B}$ is the $\mathrm{X}$-ray image obtained at $200-\mathrm{cm}$ distance from the focal point of the X-ray generator. The reference distance between the X-ray generator and the target was adjusted by using the horizontal and vertical laser system for the primary beam alignment process. From the images of the pinhole collimator which was placed at the center of the
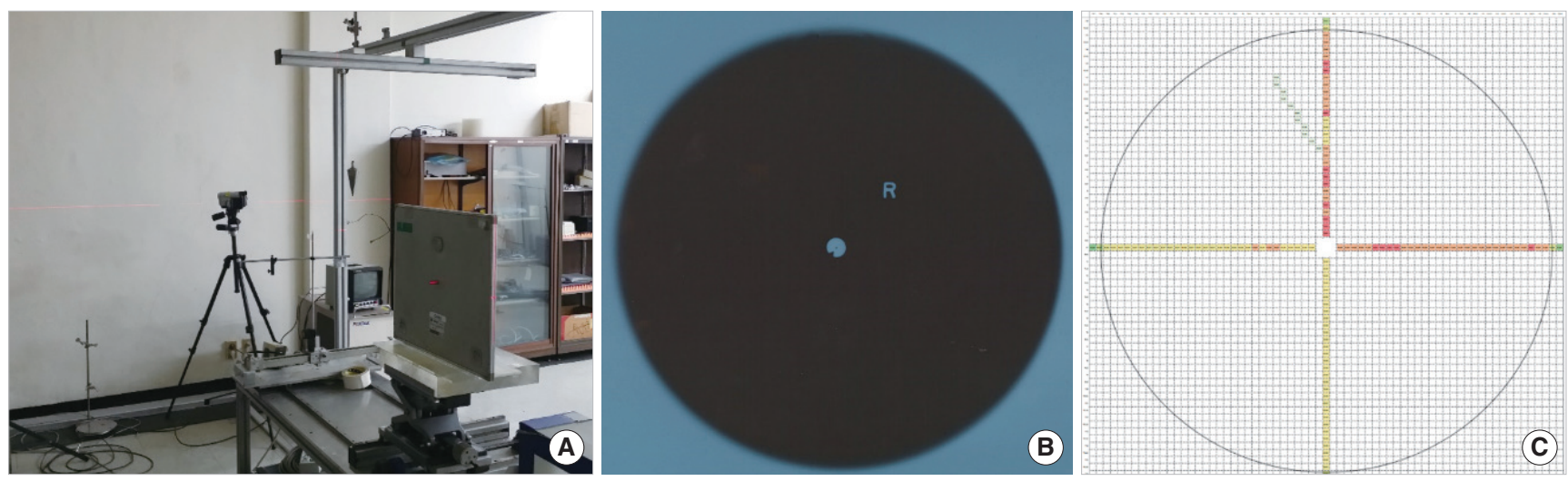

Fig. 2. (A) Beam alignment process using an X-ray film, (B) the film image acquired by the X-ray irradiator, (C) results of beam alignment using a densitometer. 
film cassette, the vertical motion of the beam at the reference distance was confirmed. The relative beam intensity was measured by analyzing the $\mathrm{X}$-ray film images from the densitometer at $5-\mathrm{mm}$ intervals.

The method using X-ray film and densitometer has a variation in the results depending on the analyst. By this method, the relative distribution of X-ray beams can be determined, but it is difficult to determine the accurate distribution of an X-ray beam. For this reason, the tertiary beam alignment process was performed for more precise measurement and analysis. The beam uniformity was measured at $200-\mathrm{cm}$ reference distance by using the Exradin A3 ion chamber (Active volume: $3.6 \mathrm{~mm}^{3}$ ). In this process, air kerma rates were measured at $1-\mathrm{cm}$ intervals using an ion chamber at the tube voltage of $60 \mathrm{kV}$ and a tube current of $20 \mathrm{~mA}$. Figure 3 is the picture of the experimental setup for the tertiary beam alignment. The air kerma rates of the $\mathrm{X}$-ray generator were measured using the ion chamber by moving it in horizontal and vertical directions from the reference laser.

\section{Half-value layer measurement}

In this study, additional filtration was introduced in order to generate X-ray beams of uniform qualities and select the beam quality from nine beam qualities including the NIST $\mathrm{M}$-series and ISO NS-series beams by rotation. Table $1 \mathrm{de}-$ scribes different types of additional filters from the NIST moderate series and those provided by KAERI. Table 2 shows the comparison of additional filters from the ISO NS-series

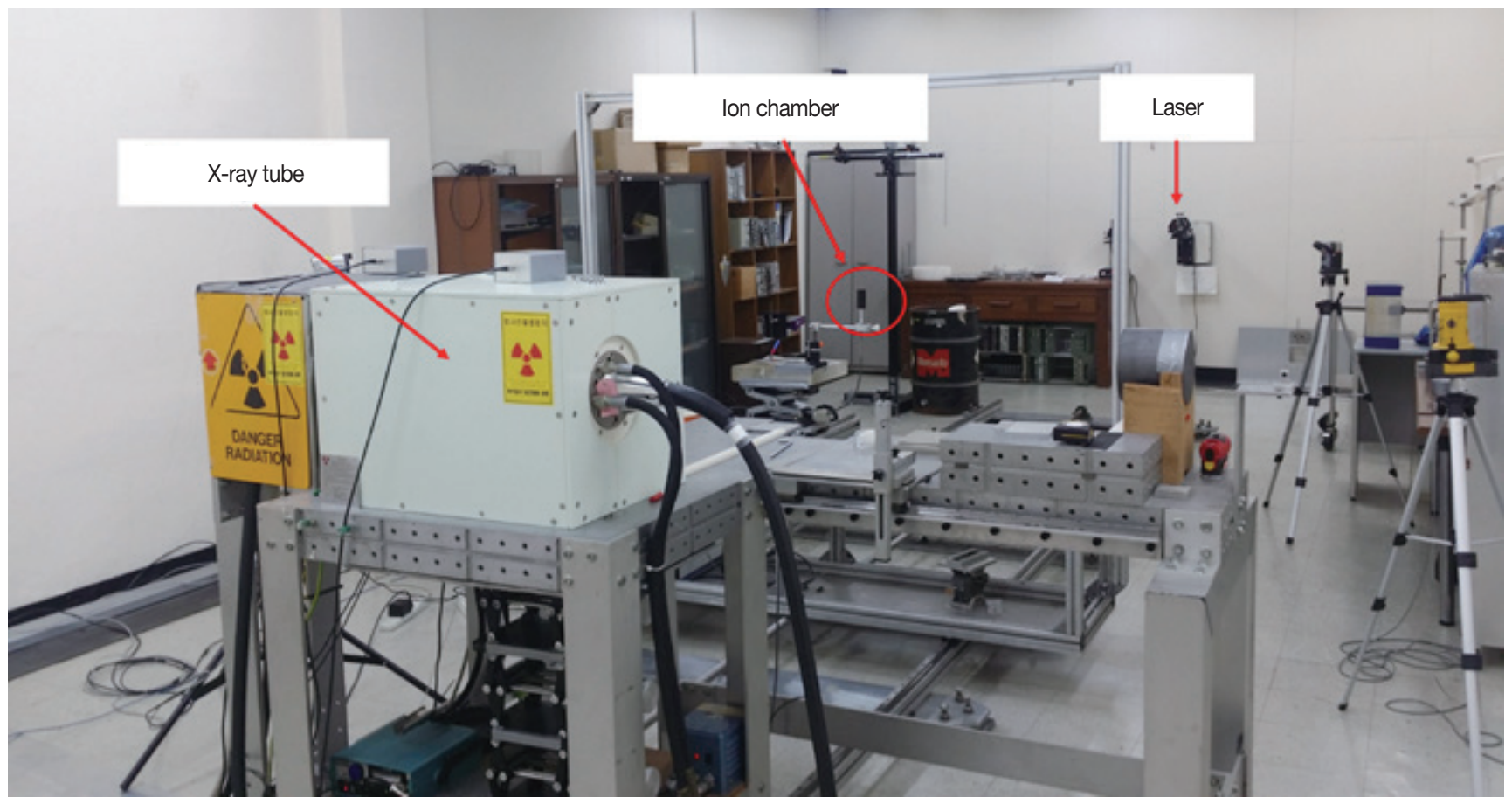

Fig. 3. Experimental setup for the 3rd beam alignment using an ion chamber.

Table 1. Results of the Air Kerma Rate Measurements Using an lon Chamber (Expressed as Relative Deviation from the Average Air Kerma Rate in Percentage)

\begin{tabular}{|c|c|c|c|c|c|c|c|c|c|c|c|c|c|c|}
\hline Distance (cm) & -16 & -15 & -14 & -12 & -10 & -5 & 0 & 5 & 8 & 10 & 12 & 14 & 15 & 16 \\
\hline 16 & - & - & - & - & - & - & 58.1 & - & - & - & - & - & - & - \\
\hline 15 & - & - & - & - & - & - & -7.6 & - & - & - & - & - & - & - \\
\hline 14 & - & - & - & - & - & - & 0.4 & - & - & - & - & - & - & - \\
\hline 10 & - & - & - & - & - & - & 0.10 & - & - & - & - & - & - & - \\
\hline 0 & 20.2 & 2.7 & -2.1 & 1.6 & 1.1 & 0.03 & 0.5 & 1.2 & 2.0 & 1.5 & 1.4 & 1.4 & 0.7 & 42.6 \\
\hline-10 & - & - & - & - & - & - & 0.1 & - & - & - & - & - & - & - \\
\hline-14 & - & - & - & - & - & - & -0.6 & - & - & - & - & - & - & - \\
\hline-15 & - & - & - & - & - & - & -7.9 & - & - & - & - & - & - & - \\
\hline
\end{tabular}


and those provided by KAERI.

To compare the X-ray beam quality produced by KAERI with the use of the existing additional filter with beam qualities specified in the international standards, HVL measurements were conducted and the results were compared with those specified in NIST and ISO-4037, as shown in Figure 4. NE2530/1 ion chamber (Active volume: $35 \mathrm{~mm}^{3}$ ) was used for the HVL measurements. A total of nine beam qualities were analyzed at a reference distance of $200 \mathrm{~cm}$. A measurement of air kerma rates for the HVL values stated in NIST and ISO-4037 was made, and values for the first and second HVLs were compared and checked to see if they conform to the values stated in NIST and ISO.

\section{Results and Discussion}

In this study, beam alignment was undertaken in three steps to deliver a uniform radiation dose to the target at different distances. The primary beam alignment was performed by physical methods using the horizontal and vertical laser positioning system to ensure that the focal point of the X-ray generator is at the center of the target. Figure 2C describes the measurement results of the X-ray film images obtained in the secondary beam alignment process by using the densitometer. The results showed that the X-ray generator generates a beam with a diameter of around $30 \mathrm{~cm}$ at the reference distance of $200 \mathrm{~cm}$. The measurement with the densitometer showed that the intensity of the X-ray beam

Table 2. Characteristics of the Moderate Filtration Series (NIST M-series)

\begin{tabular}{|c|c|c|c|c|c|c|c|}
\hline \multirow{3}{*}{ Beam code } & \multirow{3}{*}{ Tube potential (kV) } & \multicolumn{3}{|c|}{ KAERI (Inherent filtration: 3.0 mm-Be+1.592 mm-Al) } & \multicolumn{3}{|c|}{ NIST (Inherent filtration: 3.0 mm-Be) } \\
\hline & & \multicolumn{3}{|c|}{ Additional filtration (mm) } & \multicolumn{3}{|c|}{ Additional filtration (mm) } \\
\hline & & $\mathrm{Sn}$ & $\mathrm{Cu}$ & Al & $\mathrm{Sn}$ & $\mathrm{Cu}$ & Al \\
\hline M60 & 60 & - & - & - & - & - & 1.56 \\
\hline M100 & 100 & - & - & 3.67 & - & - & 5.0 \\
\hline M150 & 150 & - & 0.259 & 3.432 & - & 0.25 & 5.0 \\
\hline $\mathrm{H} 150$ & 150 & 1.51 & 4.94 & 3.93 & 1.51 & 4.0 & 4.0 \\
\hline
\end{tabular}

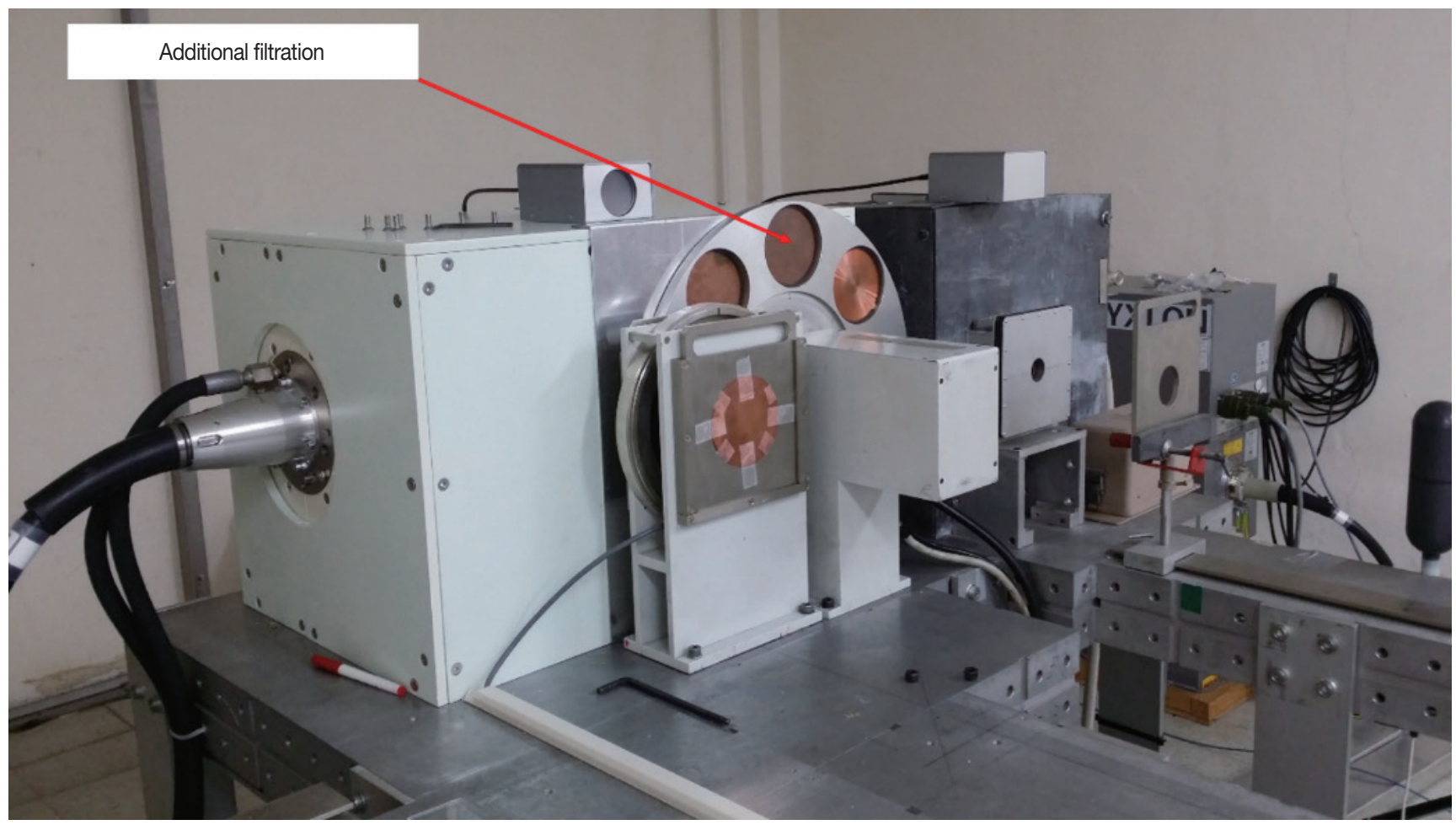

Fig. 4. The rotation type additional filtration system manufactured by the KAERI and experimental setup for half-value layer (HVL) measurements. 
ranges from 0.58 to 0.61 .

Finally, the tertiary beam alignment was performed to provide a more accurate beam distribution. Table 3 presents the results of air kerma rate measurements using an ion chamber. Each number listed in Table 3 is expressed as a percent deviation from the average air kerma rate. As shown in Table 3, uniformity of beam intensity was established with $\pm 2.5 \%$ variation of the air kerma rate, within the beam diameter of $28 \mathrm{~cm}$. This met the requirement of ISO-4037 concerning field uniformity, stating the $5 \%$ limit for variations in the air-kerma rate.

Tables 4 and 5 show the results of HVL measurements for NIST moderated series and ISO narrow spectrum series of beam and measurements by using the additional filter man-

Table 3. Characteristics of the Narrow-Spectrum Series (ISO NSseries)

\begin{tabular}{|c|c|c|c|c|c|}
\hline \multirow{3}{*}{$\begin{array}{l}\text { Beam } \\
\text { code }\end{array}$} & \multirow{3}{*}{$\begin{array}{l}\text { Tube } \\
\text { potential } \\
\text { (kV) }\end{array}$} & \multirow{2}{*}{\multicolumn{2}{|c|}{$\begin{array}{c}\text { KAERI (Inherent } \\
\text { filtration: } 3.0 \mathrm{~mm}-\mathrm{Be} \\
+3.727 \mathrm{~mm}-\mathrm{Al}) \\
\text { Additional filtration }(\mathrm{mm})\end{array}$}} & \multirow{2}{*}{\multicolumn{2}{|c|}{$\begin{array}{c}\text { ISO narrow-spectrum } \\
\text { series (Inherent filtration: } \\
4.0 \mathrm{~mm} \text { equilibrium at } 60 \mathrm{kV} \\
\text { Additional filtration }(\mathrm{mm})\end{array}$}} \\
\hline & & & & & \\
\hline & & Sn & $\mathrm{Cu}$ & Sn & $\mathrm{Cu}$ \\
\hline NS60 & 60 & - & 0.6 & - & 0.6 \\
\hline NS80 & 80 & - & 2.0 & - & 2.0 \\
\hline NS100 & 100 & - & 5.0 & - & 5.0 \\
\hline NS120 & 120 & 1.0 & 5.0 & 1.0 & 5.0 \\
\hline NS150 & 150 & 2.5 & - & 2.5 & - \\
\hline
\end{tabular}

ufactured by KAERI, respectively.

ISO-4037 states that if the difference between the first and second HVL values of two X-ray beams is within $\pm 5 \%$, then these two X-ray beams can be considered as equivalent. The allowable error in the thickness of the filters used for the HVL measurements was set to be less than $\pm 0.005 \mathrm{~mm}$, and the filter materials with purity no less than $99.9 \%$ were used. The measurement results of the first and second HVLs using an ion chamber were shown in Tables 4 and 5, and the values are expressed as percent deviations between measurements with and without the HVL. In Tables 4 and 5, the agreement ratios are expressed as a percentage, and the homogeneity coefficient represents the ratio of the first HVL to the second HVL. As shown in Tables 4 and 5, the first and second HVLs differ by a maximum of $3.72 \%$, and this met the requirement of the standard document stating $\pm 5 \%$ limit for variation between the first and second HVLs. Therefore, the beams can be considered as equivalent to the beam qualities of the NIST M-series and ISO NS-series.

\section{Conclusion}

In this study, air kerma rates were measured for the first and second HVLs to ensure the effectiveness of beam alignment and beam qualities of the new X-ray irradiation system. The beam alignment was carried out in three steps to ensure

Table 4. The Results of HVL Measurements for the NIST Moderated Series

\begin{tabular}{|c|c|c|c|c|c|c|c|c|}
\hline \multirow{3}{*}{ Beam codes } & \multirow{3}{*}{$\begin{array}{l}\text { Air kerma rate } \\
\qquad\left(m G y \cdot h^{-1}\right)\end{array}$} & \multicolumn{6}{|c|}{ NIST Moderated series beam } & \multirow{3}{*}{$\begin{array}{c}\text { Homogeneity } \\
\text { coefficient }\end{array}$} \\
\hline & & \multicolumn{3}{|c|}{ 1st HVL } & \multicolumn{3}{|c|}{ 2nd HVL } & \\
\hline & & $\begin{array}{l}\mathrm{HVL} \\
(\mathrm{mm})\end{array}$ & $\begin{array}{c}\text { Air kerma rate } \\
\left(\mathrm{mGy} \cdot \mathrm{h}^{-1}\right)\end{array}$ & $\begin{array}{c}\text { Ratio } \\
\text { (Agreement, \%) }\end{array}$ & $\begin{array}{l}\mathrm{HVL} \\
(\mathrm{mm})\end{array}$ & $\begin{array}{c}\text { Air kerma rate } \\
\left(\mathrm{mG} \cdot \mathrm{h}^{-1}\right)\end{array}$ & $\begin{array}{c}\text { Ratio } \\
\text { (Agreement, \%) }\end{array}$ & \\
\hline M60 & 619.71 & $1.673 \mathrm{Al}$ & 310.08 & 0.07 & $2.55 \mathrm{Al}$ & 152.44 & 1.61 & 66 \\
\hline M100 & 609.04 & $5.03 \mathrm{Al}$ & 308.06 & 1.16 & $6.905 \mathrm{Al}$ & 152.60 & 0.22 & 73 \\
\hline M150 & 830.85 & $10.25 \mathrm{Al}$ & 413.95 & 0.36 & $11.515 \mathrm{Al}$ & 211.19 & 1.67 & 89 \\
\hline $\mathrm{H} 150$ & 16.80 & $2.50 \mathrm{Cu}$ & 8.41 & 0.36 & $2.632 \mathrm{Cu}$ & 4.19 & 0.28 & 95 \\
\hline
\end{tabular}

Table 5. The Results of HVL Measurements for the ISO Narrow Spectrum Series

\begin{tabular}{|c|c|c|c|c|c|c|c|c|}
\hline \multirow{3}{*}{ Beam codes } & \multirow{3}{*}{$\begin{array}{c}\text { Air kerma rate } \\
\left(m G y \cdot h^{-1}\right)\end{array}$} & \multicolumn{6}{|c|}{ ISO (Characteristics of narrow-spectrum series) } & \multirow{3}{*}{$\begin{array}{c}\text { Homogeneity } \\
\text { coefficient }\end{array}$} \\
\hline & & \multicolumn{3}{|c|}{ 1st HVL } & \multicolumn{3}{|c|}{ 2nd HVL } & \\
\hline & & $\begin{array}{l}\mathrm{HVL} \\
(\mathrm{mm})\end{array}$ & $\begin{array}{c}\text { Air kerma rate } \\
\left(\mathrm{mG} \cdot \mathrm{h}^{-1}\right)\end{array}$ & $\begin{array}{c}\text { Ratio } \\
\text { (Agreement, \%) }\end{array}$ & $\begin{array}{l}\mathrm{HVL} \\
(\mathrm{mm})\end{array}$ & $\begin{array}{l}\text { Air kerma rate } \\
\left(m G y \cdot h^{-1}\right)\end{array}$ & $\begin{array}{c}\text { Ratio } \\
\text { (Agreement, \%) }\end{array}$ & \\
\hline NS60 & 30.38 & $0.24 \mathrm{Cu}$ & 14.90 & 1.89 & $0.26 \mathrm{Cu}$ & 7.67 & 0.99 & 92 \\
\hline NS80 & 18.18 & $0.58 \mathrm{Cu}$ & 9.10 & 0.17 & $0.62 \mathrm{Cu}$ & 4.53 & 0.25 & 94 \\
\hline NS100 & 7.83 & $1.11 \mathrm{Cu}$ & 4.00 & 2.17 & $1.17 \mathrm{Cu}$ & 2.01 & 2.63 & 95 \\
\hline NS120 & 9.36 & $1.71 \mathrm{Cu}$ & 4.78 & 2.09 & $1.77 \mathrm{Cu}$ & 2.42 & 3.29 & 97 \\
\hline NS150 & 63.19 & $2.36 \mathrm{Cu}$ & 32.73 & 2.64 & $2.47 \mathrm{Cu}$ & 16.39 & 3.72 & 96 \\
\hline
\end{tabular}


uniformity of beam qualities and provide a more accurate beam distribution. The beam alignment was performed by physical methods using the laser positioning system to ensure that the new $\mathrm{X}$-ray generator is aimed at the right angles, both vertically and horizontally, to the target. The final beam alignment process was performed using an X-ray film and ion chamber. As a result, the uniformity of beam intensity was established with $\pm 2.5 \%$ variation of the air kerma rate, within a beam diameter of $28 \mathrm{~cm}$, and this ensured the requirement ISO-4037 stating that field uniformity should not exceed $5 \%$. Air kerma rates were measured for the first and second HVLs stated in NIST and ISO-4037 using an ion chamber. The results showed that the difference between the values was within $\pm 5 \%$, and this met the requirements for equivalent beam qualities stated in the standard documents. In conclusion, the beam qualities of the reference X-ray field from the new X-ray generator were found to meet the specifications of the NIST M-series and ISO NS-series beam qualities. Further measurements of X-ray spectra will be conducted by using a low-energy high-purity germanium detector to compare with the mean energy stated in the international standard documents. In addition, personal dosimeter performance testing and calibration of radiation detector will be continuously undertaken by using the new reference $\mathrm{X}$-ray fields.

\section{Acknowledgements}

This work was supported by the National Research Foundation of Korea (NRF) grant funded by the Korea government (Ministry of Science and ICT) (No.2017M2A8A4015255) and the Nuclear Safety Research Program through the Korea Foundation Of Nuclear Safety (KoFONS) using the financial resource granted by the Nuclear Safety and Security Commission (NSSC) of the Republic of Korea (No.1605006).

\section{References}

1. International Organization for Standardization. $\mathrm{X}$ and gamma reference radiation for calibrating dosimeters and dose rate meters and for determining their response as a function of photon energy-Part 1: Radiation characteristics and production methods. ISO 4037-1. 1996;4-12.

2. American National Standard for Dosimetry. Personnel Dosimetry Performance-Criteria for Testing, ANSI/HPS N13.11-2009. 2009;13-18.

3. Korea Association of Standards \& Testing Organizations. Standard Calibration Procedure of Personal Dosimeter for X-ray. KASTO 17-80102-397. 2017;1-10.

4. YXLON Technical data. Y.TU 320-D01/D03/D05.

5. National Institute of Standards and Technology. NIST measurement services: Calibration of X-ray and gamma-ray measuring instruments. NIST Special Publication. 2001;250-258. 